\title{
Construction of Intelligent Transportation Network Data Based on Open Street Map
}

\author{
Wang Danping ${ }^{1,2,3}$, Hu Kunyuan ${ }^{1}$, Han Xiaowei $^{2}$ \\ ${ }^{1}$ Shenyang Institute of Automation Chinese Academy of Sciences, 110016 Shenyang, China \\ ${ }^{2}$ Shenyang University, 110044 Shenyang, China \\ aiamdanping@163.com
}

Keywords: Open Street Map; Intelligent transportation management; Road-net Data DCR.

\begin{abstract}
With the rapid development of economy and society, the city scale continues to expand while transportation is under great stress. Constructing the intelligent transportation system is an effective solution to improve the level of transportation management and decision support. Against the background of the applications of ITS, the paper investigates the construction processing and conversion based on OSM road-net data. It mainly explores the construction process, reconfiguration and fusion of OSM data which helps making the road\}et data independent on the application system under the background of huge amounts of city calculations and realizing the aim of standardization in road-net data semantic. Additionally we still study several problems that probably occur during the construction and reconfiguration process of road-net data in order to avoid unreasonable data construction and to satisfy the demands of intelligent city administration for the reliability and stability of the basic data.
\end{abstract}

\section{Introduction}

Intelligent Transportation Systems (Intelligent Transportation System, ITS) is a complex distributed system, the integration of communications, sensing, mobile positioning, databases, intelligent information processing, automatic control and other areas of advanced technologies designed to improve transportation efficiency and safety reduce traffic accidents, reduce environmental pollution, in order to establish a smart, safe, convenient, efficient and environmentally friendly integrated transport system. Intelligent transportation systems generally include traffic management, traveler information, public transport, vehicle control, transportation management, electronic toll collection, emergency and other security features.

Domestic and foreign construction of intelligent transportation systems, roads, vehicles, lights, cameras continue to improve transport infrastructure, sensing, wireless communication, intelligent terminals, Internet, cloud computing and other IT get utilization, transportation information platform has accumulated more An increasing number of traffic data, and to promote the application of intelligent transportation technology in public transport systems, integrated transport and so on. Intelligent transportation industry under in a great opportunity for development, and will continue to show high growth trend over a longer period of time [1].

Intelligent transportation system construction must be based on intelligent transportation systems framework. Framework of intelligent transportation system is mainly based on the needs of users in a geographical area, the definition of system functionality, content and methods of information exchange between functional domains, each function processing, etc., for all levels of information sharing, exchange and co-processing specification, is a qualitative description of the intelligent transportation system is to ensure that the process of coordination and technical integration architecture construction and implementation of intelligent transportation systems.

The main advantage of real-time traffic data collection mobile positioning, wireless sensing, graphic images, and other wireless communications technologies quickly and accurately target location, detection, identification and transmission, is to achieve traffic management, basic traffic guidance, intelligent control, traffic information service. Over time, more and more of the original data can be brought together through the traffic integrated information platform. These data are not 
only used for traffic management, control, real-time applications such as induction, more importantly, can extract implicit knowledge to aid decision making. Traffic management and traveller decision support capabilities are all intelligent transport systems put a higher demand, in which dynamic traffic and road network traffic congestion state analysis is performed intelligent analysis and decision support for important content.

\section{OSM and road network data}

Compared with other geospatial data, OSM biggest feature and advantage of the openness of the map data such as the open road network data characteristics similar to open Wikipedia content: seeds from global volunteer network data based on their geographical location known, the use of the road network of GPS tool basic physical data acquisition, data .OSM construct open road network data including the timeliness and accuracy of coexistence, the speed and the data is completely open its atomic data intact solved research personnel and processes for application developers to work for the development of work provides valuable resources. with the gradual improvement of the road network OSM data, OSM road network data can be layered to achieve more likely to provide a wealth of navigation information for the modern urban residents, such as driving, walking, cycling, horseback riding, etc. have been providing a personalized service. Therefore, analysis, discussion and research OSM's road network data, to promote the development of geographic information systems for modern fast pace of life to provide efficient road network information services have a very important academic and practical significance [2].

OSM data primitives include: layers (Layers), points (Nodes), road (Ways) and relationships (Relations) have research data shows that layer and the relationship does not belong to the OSM data primitives layer is applied research or certain. some scientific relations defined; whereas the relationship just a primitive description of certain correlation properties so that the first two data constituting the atomic primitive data OSM data POI data and city streets, while layer and relationships define the relationship between logical application topology and logical storage elements. As a research and sharing the road network for the purpose of spatial data, attribute values OSM data primitives are very rich, which means that the full expression of spatial data as possible in order to facilitate data users in different areas of extraction.

OSM spatial database with traditional databases, require the physical layer has a special structure to support the search operation, such as point queries, range queries, therefore, spatial database logic layer using a non-standard database management approach to deal with the complexity of spatial data, dynamic, there is no space algebra standardized features multidimensional space operation is not closed and spatial data, etc. However, since it is a logical combination of point sets, we will follow the point sets relational features, therefore, OSM road network data and other GIS data, the storage and logical relations is a vector relationship, points, lines and objects also include external attributes of objects, spatial position of the object with the object of expanding and therefore the relationship between the system network topology data is point-to-point primitives and primitives, primitives and path point primitives, primitives correlation path and the path between the primitives [3]. Fig.1 shows the relationship between the data and the attributes of the description.

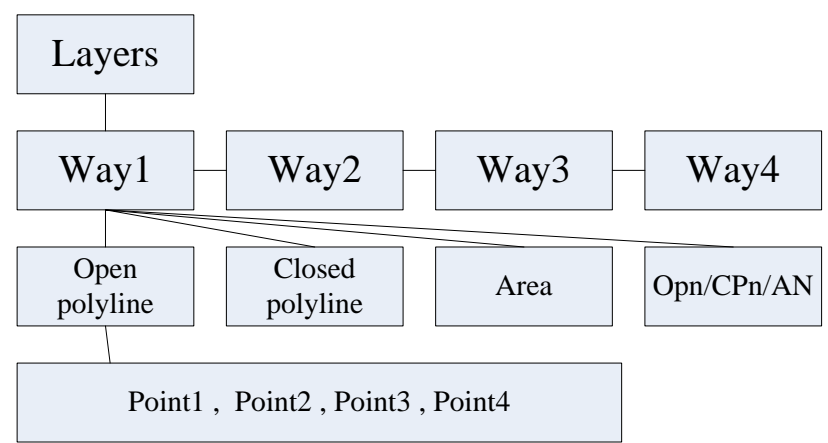

Fig. 1.The relationship between the data and the attributes of the description 


\section{Spatio-temporal data mining}

Data Mining ((Data Mining, DM) is to focus on large amounts of data to extract hidden from the process and potentially useful knowledge in the field of artificial intelligence and information science, knowledge can be represented as a concept, rules, laws, patterns and other forms of user interest. common data mining methods include clustering, association rules, prediction, anomaly detection, etc. in fact, data mining is a process of knowledge from the data structure to structure conversion, the conversion process, there should be no new knowledge generation, changing only the encoding data-driven data mining is to generate knowledge and learning through the data used to solve practical problems. You can get direct manipulation of data-driven thinking, application of knowledge, and to provide an appropriate response to a particular action or standard tasks. In order to ensure the data mining process invariance knowledge necessary to select some of the features in the data, and use these features to control the data mining process.

Intelligent traffic data analysis and decision support intelligent transportation system construction important content, which, dynamic traffic and road network congestion state analysis is focused and effective organization and management of traffic data and traffic data mining is a dynamic analysis of traffic flow and road network two key aspects of the congestion status of the analysis. Traffic data organization and management, which, depending on the interest and demand for services and traveler traffic management department, the traffic data of different granularity of abstraction, temporal and semantic features extracted, the definition of a scientific and reasonable data structure to determine how the data is stored and typical operation, and use of database technology for effective management. Traffic data organization and management is the basis for traffic data analysis [5].

Temporal data mining (Spatio Temporal Data Mining, STDM) refers to extract implicit knowledge in temporal databases, thus revealing the inherent laws of time and space in the data, internal relations and trends. Temporal data mining knowledge involves temporal databases, data mining, spatial visualization and other areas, and quickly tap the space-time quantitative knowledge bottleneck problem is temporal data processing and analysis. Spatio-temporal data mining can be into temporal data preparation, data mining, temporal, and other temporal knowledge interpretation and evaluation. Unlike traditional data mining, spatiotemporal data mining process requires consideration of temporal constraints between data and information. Temporal data preparation mainly refer to the acquisition time, spatial and attribute information data extracted temporal relationship between the data and the data is organized and stored in accordance with the user's interest. Temporal data usually has the dynamic, multi-source heterogeneous characteristics, require complex data conversion, information fusion processing during data preparation.

Traffic data analysis and mining, which according to the traffic management and traveller access to real-time traffic information and intelligence requirements to dynamic traffic and road network congestion status-oriented analysis, combined with spatial and temporal characteristics of data traffic and transportation constraints, depth analysis of the potential similarities between correlation and correlation data, and traffic data clustering analysis, predictive analysis, correlation analysis, anomaly detection depth of excavation, to discover different characteristics dimensions, different data granularity implicit knowledge provide decision support for traffic management and traveller. Traffic data analysis and data mining is the core of traffic analysis. Temporal data analysis is usually for specific areas or for a specific problem. This article is for intelligent transportation, demand for dynamic analysis of traffic flow and road network congestion status to the road network and moving objects as the main basis of analysis the road network topological and temporal characteristics of the traffic flow on the design concept model of dynamic transportation network, integrated spatial data management and streaming data management technology, multi-temporal data granularity hierarchical organization and management, support for user access to the track, traffic, and status of different data elements [6]. 


\section{The key technologies of OSM}

The construction of road-net data for Intelligent Transportation System(ITS) based on Open Street Map(OSM) are the foundation and precondition of realizing the calculation of the ITS. This method considers the spatial and temporal constraints simultaneously, which filters irrelevant data in advance and improves the efficiency of spatio-temporal association rule discovering. Based on the idea, there is analysis of the time validity and spatial relativity simultaneously during the generation of frequency item sets. It classifies the time duration of spatio-temporal data and considers the spatial relationship firstly and generates the transaction table, then performs join operation on spatial-related item sets. Experiments illuminate that the algorithm is well performed. The algorithm is applied in intelligent transportation spatio-temporal system to analysis the trend of traffic congestion by association between road segments. The key technologies of OSM are as following [7]. Fig.2 shows the key technologies of OSM.

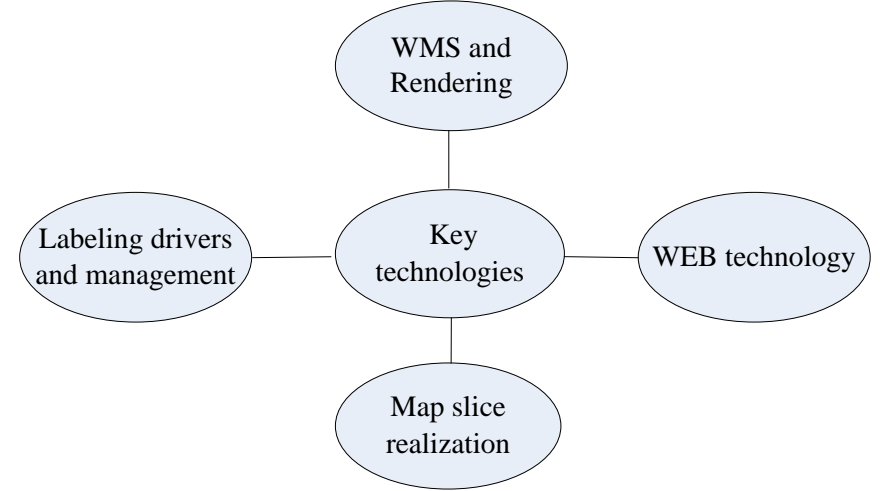

Fig. 2.The key technologies of OSM

The purpose of the road network data applications, in addition to a small number of scientific studies comparing data, part of the city and traffic management for, and most of it is developed into a WEB application. Therefore, the data provided by the server follows the W3C specification protocol conversion into an XML file format. Thus to achieve OSM service primitive data is driven by the simple tag (Tag) management map label is not an essential element, but is defined by OSM tag (tag), information data recording and integration. As many of the key or the value of the data to be recorded at the same time, we can use an additional namespace to add additional information with labels to describe the place names, road type and other information in order to achieve the management of geographic data to facilitate research and development data .

Slicing technology is provided by the system tile technology for dividing a big map into many small squares (see below). In the loaded map, just load this region correlation within a small square put out on it. Map tiles are usually 256 x 256 pixels, although this is not certain, but because of Google Map, which in fact has become the de facto standard. This standard no doubt for OSM in the effective use of technology in the field of promotion and increased resources operability.

Mature image service technology is a major feature of OSM, but also for the technology rapidly WEB applications and GIS applications reflect their own advantages. Compared to some other are GIS relatively strong data and technology systems, OSM lightweight the data structure and the conversion function is its advantage, OSM map provided by three major technology: Web map Service (WMS): is the abbreviation of web Map image service which is in line with the OGC standard HTTP; the navigation map. Open Street Map compared to more personalized service features from the layer data structure when the unlimited expansion and innovation, such as the energy for driving, traffic guidance and other areas to provide corresponding navigation information. Rendering (Rendering) technology can render the original geographic data visualization map rendering process. Rendering technology can achieve the OSM open road network data through WEB and Internet services for the world as possible. Besides, the technology can also assist containing images drawn map tile production or vector, 3D type map production [8]. 


\section{Construction and reconstruction of the road network data in the OSM}

OSM data construction process, as scientific research and applied research in atomic data for accuracy and detail of data are required as high as possible. The road network data and POI data are only at the macro as data relative to the reference from Baidu and Google. in addition to the abovementioned seed data outside of public property, in order to ensure the quality of the data, including data OSM POI Road network data and the city needs to prepare for field data collection, road network data is a collection point set, So in the process of collection, the most important is the value of the coordinate data collection of GPS data samples can provide data in the construction process will inevitably unsatisfactory even some data errors, unnecessary noise data, to follow data processing a great deal of trouble in a relatively very familiar with the terrain, the paper, the value of the work site tables of data samples, using a different coordinate representation.

For OSM most unfamiliar urban and rural road network data collection, or as good as detailed geographical markers city roads, research needs to be configured with spatial data objects with the track list of auxiliary data, usually in the order of points achieved stage depicts, in order to ensure the route changes in the relative orientation of the reference point is not obvious changes in sections [9]. Fig.3 shows the road network data and track the process of staging a road block.
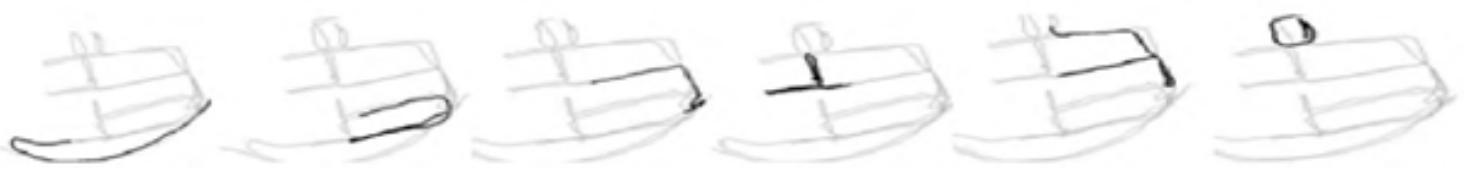

Fig. 3.The road network data and track the process

OSM road network data structure with OSM's attention improved road network based on OSM data structure platform is also increasing in the relevant field of research and the promotion of its data using open data sharing Open Database License (ODbL) authorization, data acquisition and construction the aim is to export to Open Street Map XML data structure currently provides platforms and supporting technology has become increasingly diverse, but as research data, is generally provided by Potlatch platform constructed from aerial images OSM .Potlatch for drawing large structure regional maps and rapid changes, the details of the GPS data processing as convenient, but not control the raw data collected from Baidu or Google, which in OSM openness and rigor is not consistent. Construction process, the macro-structure of the platform graphics micro data and servers in the data area needs simultaneously. Fig. 4 shows the Potlatch experimental data structure.

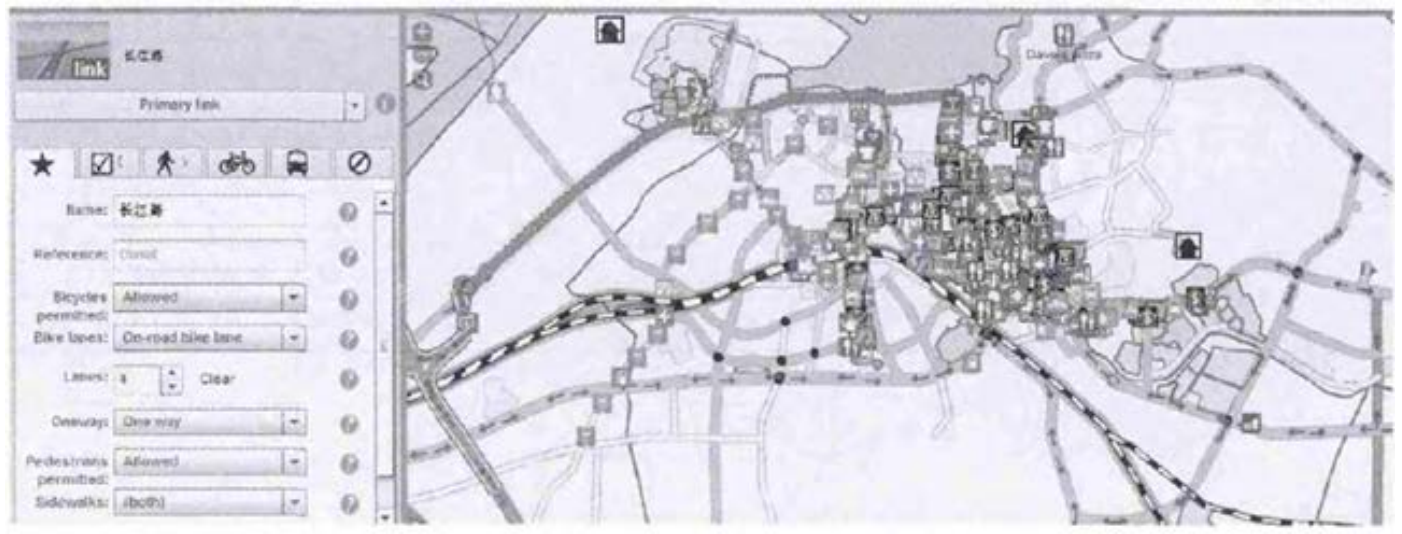

Fig. 4.The Potlatch experimental data structure

In general, all the data extracted from the system, because the check standard is objective, so basically the entire system can't detect the data overall, but the characteristics of the road network data more obvious, the road construction process data in a variety of errors, such as : two-way street only a one-way road marked; road junction incoherence; intersection incomplete data, resulting in the latter part of the calculation of the SPARQL query data is incorrect; when the data extraction area is too large, detailed road network in XML data conversion unsuccessful; part of a larger point and path coordinates marked deviation in the reconstruction process, for error and bias, it because 
of the large range of data error randomness, so only manually reconstructed to meet the basic requirements for data applications. Apart from the obvious data need to be revised, the deviation between the data path errors are important and difficult to correct [10].

When building the data, we need to engage in a review of the different layers of data, data on the bite point unreasonable reconstructed micro-data, data reconstruction principle last GPS acquisition. After repeated comparisons with some data amended to complete the construction of road network data specified range. Because of the openness of OSM system, the source data are volunteers from all over the world, in addition to incomplete data, the basic data for the study, the accuracy of its data, and it is difficult to be guaranteed. In this study, data from the sample tests from the start, with the data of a road, for example, with reference samples or data and the new data structure comparison methods such as the path between the reference sample for testing. Therefore, the data in the study area within the range of , the work is not only the basis of data engineers work to build the new data is more important and complex reconstruction and revision of data. Fig.5 shows the reconstruction and revision of data.
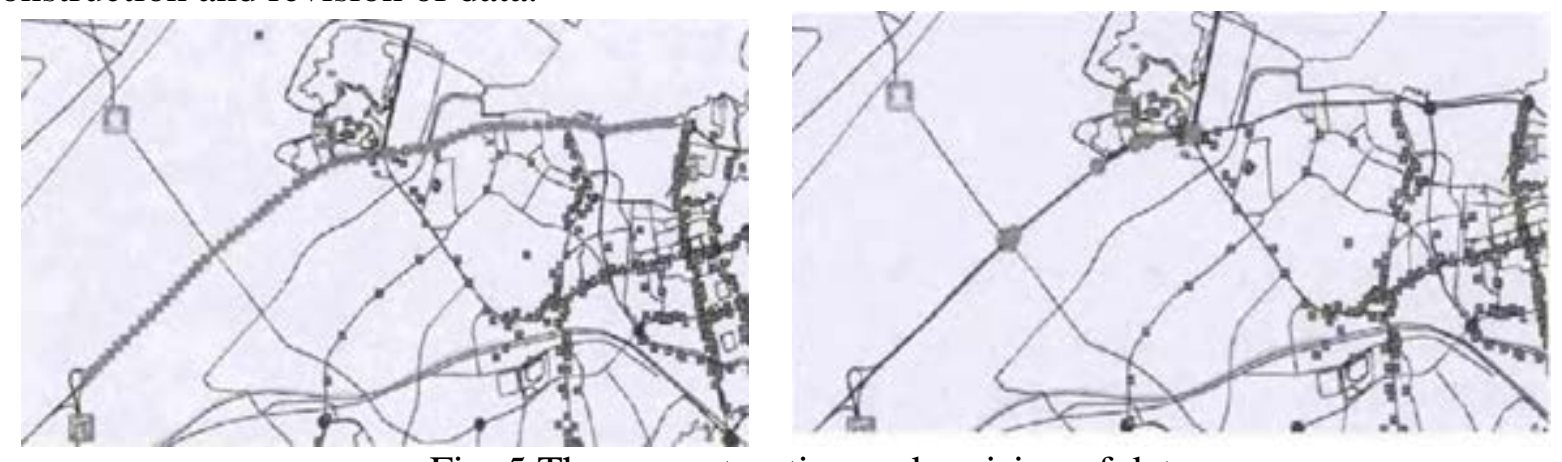

Fig. 5.The reconstruction and revision of data

\section{Conclusions}

With the rapid development of intelligent transportation systems, the ITS application data traffic network technology gets more and more attention. Intelligent transportation network data technology research and development on China Intelligent Transportation Systems is urgently needed. In this paper, the road network data structure is proposed based on OSM data of road network data to support the changing urban construction and management of information and data requirements. OSM road network construction process based on the data, the data structure of the process, the reconstruction possible problems and road network data were discussed, and accommodate basic road network data is calculated under the background of massive urban road network data independent of the system. displayed in the process of construction and reconstruction of difficult issues outlet network data structure, but also the data as much as possible to avoid unreasonable data to build in order to more effectively achieve reliability and stability of the smart city management needs of the underlying data.

\section{Acknowledgements}

The research work was supported by Liaoning city of Shenyang province science and technology plan project number: F13-298-1-00.

\section{References}

[1] Guoyin Wang. Introduction to 3DM: Domain-Oriented Data-Driven Data Mining[C]. RSKT 2008, 2008: 25-26.

[2] Zhanquan Sun, Yinglong Wang, Jingshan Pan. Short-term Traffic Flow Forecasting Based on Clustering and Feature Selection[C]. Proceedings of International Conference on Neural Networks, IEEE, 2008: 577-583. 
[3] Fan Wang, Guozhen Chao Deng, Zhu Tian. Real-time Traffic Flow Forecasting Model and Parameter Selection Based onSVR[C]. Proceedings of International Conference on Intelligent Control and Automation, IEEE, 2008:2870-2875.

[4] Xia Ying, Hong Mei Gan, Gyoung Bae Kim. Design and Implementation of Advanced Traffic Monitoring System Based on Integration of Data Stream Management System and Spatial DBMS[J]. Journal of Korea Spatial Information System Society, 2009, 11(2): 162-169.

[5] Anand Ranganathan, Anton Riabov, Olivier Verscheure, Haris Koutsopoulos, Mahmood Rahmani, Baris Giis. Real-time Traffic Information Management Using Stream Computing[J]. Data Engineering, 2010, 33(2): 64-68.

[6] Oje Kwon, Yong-Soo Song, Jae-Hun Kim, Ki-Joune Li. SCONSTREAM: A Spatial Context Stream Processing System[C]. International Conference on Computational Science and Its Applications (ICCSA), 2010: 165-170.

[7] LEE T K, PARK S, HUANG Z, et al. Toward Seoul road sign management on LarKC platform [C]//Proceedings of the 9th International Semantic; Web Conference (ISWC2010), Track of Posters and Demos ,2010.

[8] Wang Yu-Ping, The Theory and Method of Evolutionary Computation, Beijing: Science Press, 2011.

[9] LI N, HUANG Z, XU D, et al. Interface Design of Semantic; System for Road Sign Management [C], Web Information Systems Engineering-WISE 2013 Workshops Springer Berlin Heidelberg, 2014:452-460.

[10] WANC D, HUANG Z, LIU Q, et al. Using Semantic Technology for Consistency Checking of Road Signs [C]. Web Information Systems Engineering-WISE 2013 Workshops Springer Berlin Heidelberg, 2014:452-460. 